\title{
New light shed on the anticancer effects of zoledronic acid
}

According to new research published in Prostate Cancer and Prostatic Diseases, zoledronic acid-an osteoclast-targeting bisphosphonate that prevents loss of bone mass-directly inhibits prostate cancer proliferation and motility, without disrupting interactions between the tumor and the vascular wall.

Previous preclinical studies have reported anticancer activities of zoledronic acid on various cancer cell lines, but have failed to establish whether the mechanism behind this growth inhibition is cytostatic or apoptotic. Data regarding the effects of this drug on cell motility and adhesion are particularly sparse.

In the study by Mani and colleagues, zoledronic acid had a significant dosedependent inhibitory effect on PC3, DU145 and LNCaP cell growth. Apoptosis (either early or late) was evident in all cell lines, but only after $72 \mathrm{~h}$ of high-dose drug pretreatment-suggesting that apoptosis is not primarily responsible for drug-induced growth inhibition.
Pretreatment of cells with zoledronic acid also resulted in decreased tumor cell migration and invasion, but did not affect cell attachment to vascular endothelium

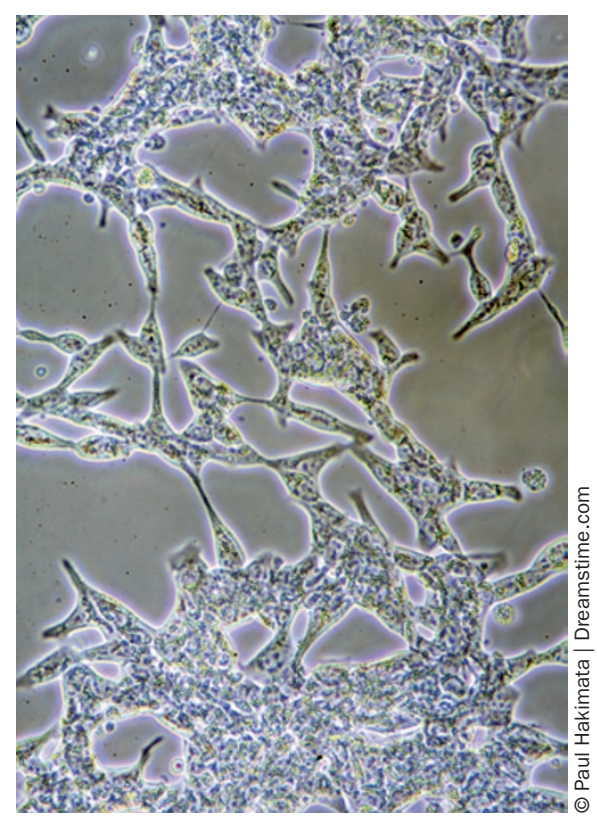

or immobilized collagen, laminin, or fibronectin (except at high doses). In PC3 cells, low-dose therapy actually enhanced adhesion to some extracellular proteins.

Mani et al. also revealed some important effects of zoledronic acid on intracellular signaling. Although drug treatment had no effect on the expression of adhesion kinases, it was associated with reduced expression of cyclin $\mathrm{E}$ and $\beta 4$-integrin, and elevated levels of p21, p27 and $\beta 1$-integrin.

Zoledronic acid is commonly prescribed to men with advanced prostate cancer and bone metastases to reduce fracture risk and treat cancer-related hypercalcemia. These data suggest that the benefits of treatment could extend far beyond these indications.

\section{Melanie Clyne}

Original article Mani, J. et al. Zoledronic acid influences growth, migration and invasive activity of prostate cancer cells in vitro. Prostate Cancer Prostatic Dis. doi:10.1038/ pcan.2012.9 